\title{
Retroperitoneal dermoid cyst mimicking a liposarcoma based on imaging assessment: case report and literature review
}

\author{
Xiaomu Ma ${ }^{1}$, Jianchun Xiao ${ }^{2}$, Weibin Wang ${ }^{2}$ \\ ${ }^{1}$ Plastic Surgery Hospital, Peking Union Medical College, Chinese Academy of Medical Sciences, Beijing, China; ${ }^{2}$ Peking Union Medical College \\ Hospital, Peking Union Medical College, Chinese Academy of Medical Sciences, Beijing, China \\ Correspondence to: Weibin Wang. Peking Union Medical College Hospital, Peking Union Medical College, Chinese Academy of Medical Sciences, \\ Beijing, China. Email: wwb_xx@163.com.
}

\begin{abstract}
Dermoid cyst is a kind of mature cystic teratoma that contains only one germ layer. It usually occurs in the head and neck, retroperitoneal dermoid cysts are uncommon. Teratomas are characterized by mixed density and features like calcification or air-fluid level. Here, we present a case of 40-year-old female with a $12.1 \mathrm{~cm} \times 10.0 \mathrm{~cm}$ retroperitoneal fatty mass which showed no calcification and few other specific characteristics of teratoma. Thin and uniform separations were observed. On PET-CT, it was adherent to several retroperitoneal organs like left adrenal gland and left kidney, and had a close relationship with several retroperitoneal vessels like aorta, superior mesenteric arteriovenous, left superior renal artery and inferior vena cava. On PET-CT, mild ${ }^{18}$ F-FDG uptake was observed. Based on the above imaging findings, a clinical diagnosis of liposarcoma was considered. After laparotomy and open surgery transit through a left vertical incision, the histopathologic examination revealed a retroperitoneal dermoid cyst. During a 2-month followup, the patient recovered well without discomfort and recurrence. Comparison between dermoid cyst and liposarcoma in imaging findings was performed. In dermoid cysts, there can be thin and uniform separations, rather than linear, localized and irregular high density in liposarcoma. The difference between dermoid cyst and mature cystic teratoma is yet to be clarified. A review of clinical and histopathological features of retroperitoneal dermoid cyst was also performed to enhance the level of the diagnosis and management.
\end{abstract}

Keywords: Case report; retroperitoneal dermoid cyst; mature cystic teratoma; liposarcoma; imaging

Submitted Jan 30, 2021. Accepted for publication Apr 16, 2021.

doi: $10.21037 /$ gs-21-65

View this article at: https://dx.doi.org/10.21037/gs-21-65

\section{Introduction}

Dermoid cysts, also known as mature cystic teratoma, are characterized by the presence of tissue from only one germ layer (1). Teratomas often occur in gonad, and extra-gonadal teratomas account for about $1-11 \%$ of retroperitoneal neoplasms (2). There were $84 \%$ of dermoid cysts reported occurring in the head and neck (3). Retroperitoneal dermoid cysts are very rare and experience in diagnosis is insufficient. Without specific characteristics of teratomas like calcification and air-fluid level in imaging, it is challenging to differentiate dermoid cysts from other retroperitoneal fat components, such as liposarcoma. We reported a rare case of retroperitoneal dermoid cyst which lacked the classical characteristics of teratomas in imaging, and was misdiagnosed as liposarcoma. Comprehensive clinical features and imaging characteristics of dermoid cysts and liposarcoma were analyzed. A literature review was also presented to improve the understanding of retroperitoneal dermoid cysts and to reduce the likelihood of misdiagnosis.

We present the following case in accordance with the CARE reporting checklist (available at https://dx.doi. org/10.21037/gs-21-65).

\section{Case presentation}

A 40-year-old female was admitted to our clinic for the operation of a retroperitoneal mass incidentally discovered 

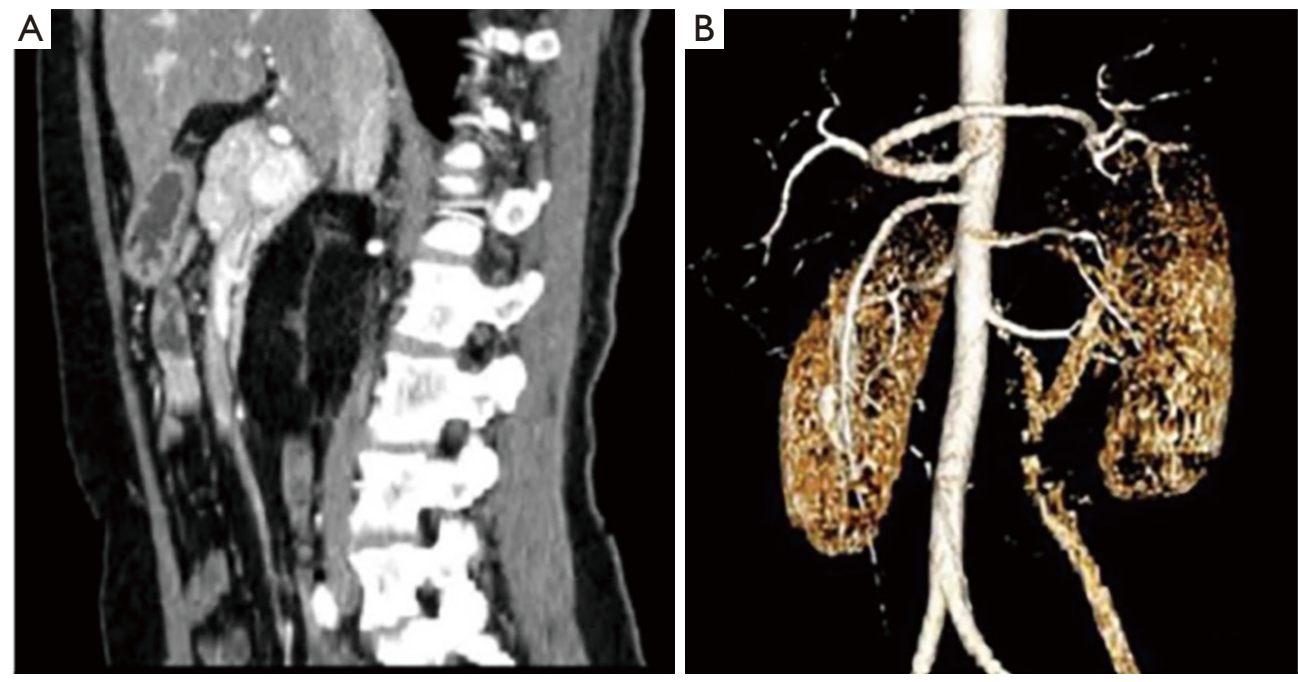

Figure 1 Image of enhanced computerized tomography (CT). (A) In the sagittal plane, a large tumor was posterior to the pancreas and duodenum which were compressed and pushed forward. Thin and uniform separations were observed. (B) Three-dimensional reconstruction showed that the lesion was behind the superior mesenteric arteriovenous.
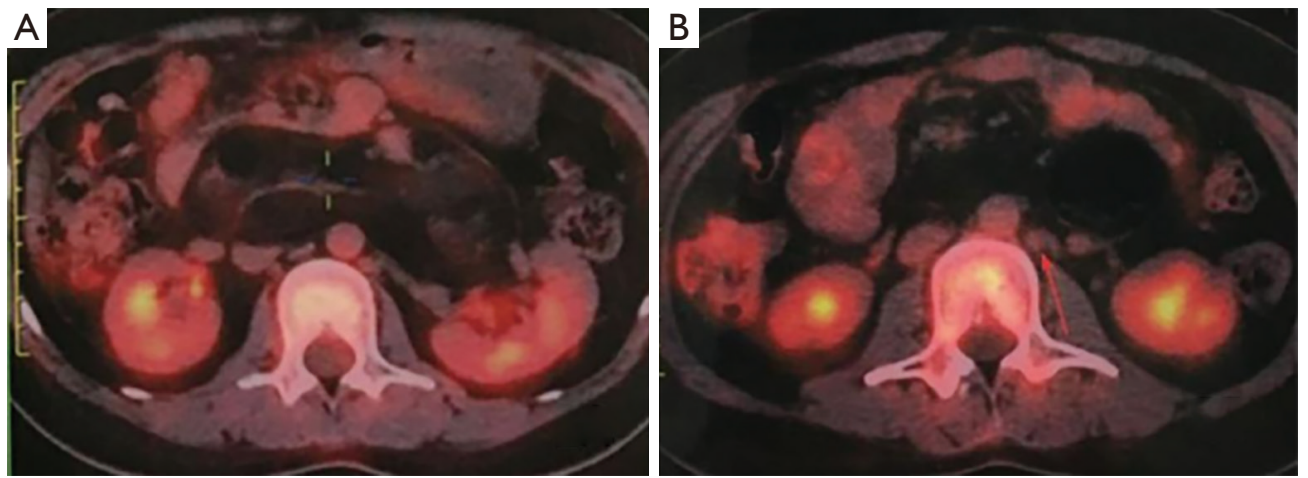

Figure 2 Image of positron emission tomography (PET)/computed tomography (CT). Lesion was encasing the aorta and the left superior renal artery, and displacing the inferior vena cava. (A) Mild ${ }^{18} \mathrm{~F}$-fluorodeoxyglucose $\left({ }^{18} \mathrm{~F}\right.$-FDG) uptake was observed in the neoplasm (white lines). (B) Mild ${ }^{18}$ F-FDG uptake was observed in the retroperitoneal lymph nodes (red arrow).

2 months ago. The medical, family and psycho-social history were unremarkable. She was asymptomatic, and physical examination showed a slight distention of the abdomen with no other obvious positive signs. The results of biochemistry and tumor markers including CA19-9, CA12-5, CA24-2, CA72-4, CA15-3, CEA, AFP were within normal ranges.

According to imaging studies, including CT and MRI, she was found to have a retroperitoneal $12.1 \mathrm{~cm} \times 10.0 \mathrm{~cm}$ mass showing fat density, irregular morphology and unclear boundary. There were hyper-density, nodular and enhanced soft tissue components inside. A three-dimensional reconstruction CT was performed and revealed a large tumor posterior to the pancreas and duodenum which were compressed and pushed forward (Figure 1). Thin and uniform separations were observed (Figures 1A,2A). Lesion was behind the superior mesenteric arteriovenous (Figure 1), encasing the aorta and the left superior renal artery and displacing the inferior vena cava (Figure 2). It was adherent to the left adrenal gland, left kidney, left renal pelvis and surrounding vessels including proximal superior mesenteric artery, left and right renal artery, and root of bilateral reproductive vein. Obviously thick and round reproductive veins were shown on both sides. Mild 


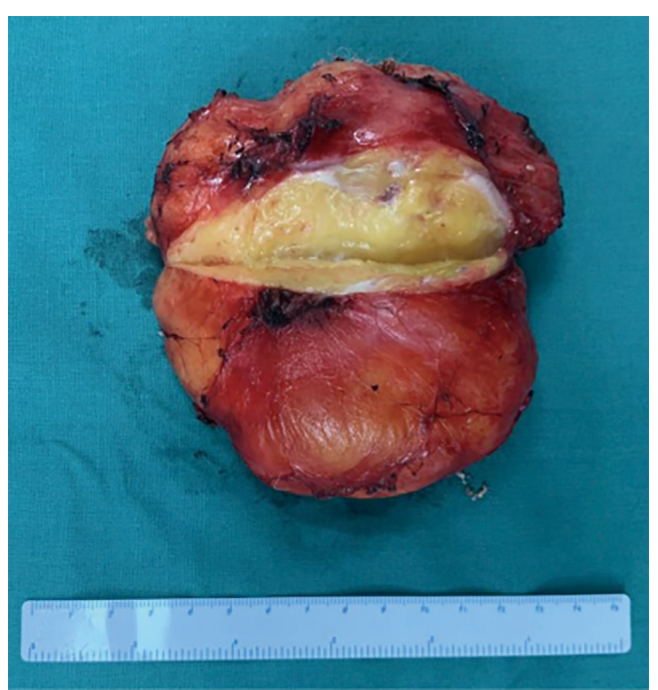

Figure 3 On gross examination, the mass was composed of fat components with greasy secretions and hair. The surface was intact.

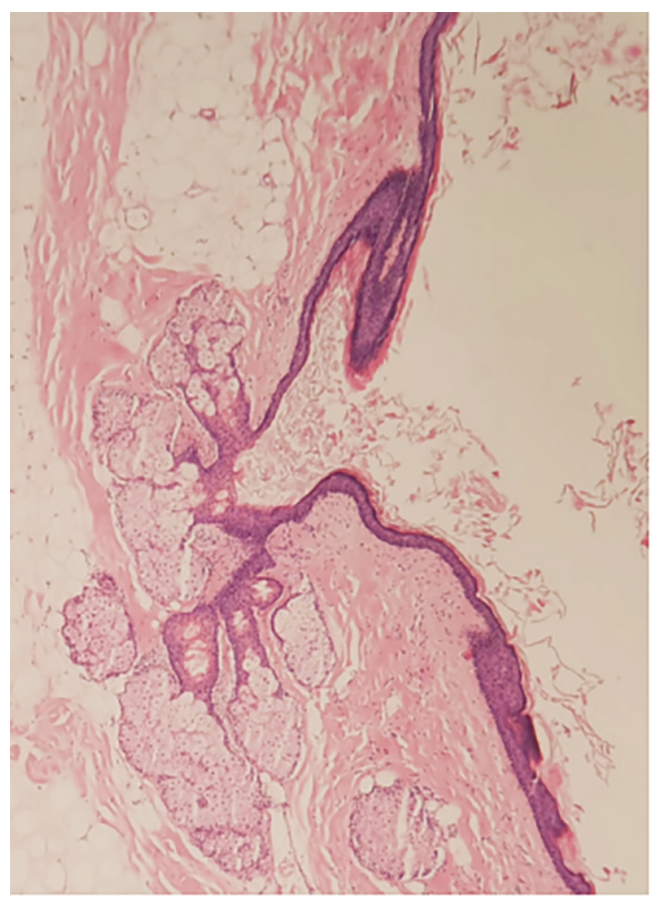

Figure 4 On histopathologic examination (Haematoxylin and Eosin, $\times 40$ ), the cyst was lined by squamous epithelium with keratinized materials and skin appendages including sebaceous gland and hair follicles. It was pathologically considered as a dermoid cyst.
${ }^{18} \mathrm{~F}$-fluorodeoxyglucose $\left({ }^{18} \mathrm{~F}\right.$-FDG) uptake was observed in the neoplasm (Figure $2 A$ ) as well as the retroperitoneal lymph nodes (Figure 2B).

A needle biopsy was difficult to perform because of the deep position in retroperitoneal space. For retroperitoneal fatty or cystic neoplasms, the differential diagnosis includes lipomyoma, liposarcoma, lymphangiocyst, bronchogenic cyst, cystic mesothelioma, cystic teratoma and so on. Except for liposarcoma, all other lesions are mostly benign without increased ${ }^{18} \mathrm{~F}$-FDG uptake in PET-CT. In addition, liposarcoma is the most common lesion, especially in adult. Teratomas usually present with calcification, and mainly occur in children and adolescent. Combined with the imaging features, location, incidence, and age of the patient, a primary diagnosis of liposarcoma was considered. The patient had been to three hospitals in total, and transferring to a higher authority hospital was always recommended due to the difficulty of the therapy. She was eager to have an operation.

The patient was informed of the possibility of incomplete tumor excision, left adrenal and left kidney resection, and high risk of recurrence of liposarcoma. Coupled with the relatively young age and absence of symptom, the option of expectant treatment was also provided. The patient finally chose surgical treatment. Bilateral Double J tubes, ureteral stents which were used to identify and protect the ureter, were implanted before surgery. Laparotomy and open surgery transit through a left vertical incision were then performed. The neoplasm was at the posterior of the pancreatic and duodenum with an intact capsule. When the left vertical incision was performed, the left renal hilum area was fully exposed and protected. The neoplasm was excised entirely.

The macroscopic examination revealed a mass composed of fat components and 2 cystic cavities, with a complete capsule (Figure 3). Greasy secretions and hair were observed. Microscopically, the cyst was lined by squamous epithelium with keratinized materials and skin appendages including sebaceous gland and hair follicles (Figure 4). The tumor was pathologically considered as a dermoid cyst. No obvious complications occurred. During a 2-month followup, the patient recovered well without discomfort and recurrence.

All procedures performed in studies involving human participants were in accordance with the ethical standards 
of the institutional and/or national research committee(s) and with the Helsinki Declaration (as revised in 2013). The study was approved by Ethics Committee of Peking Union Medical College Hospital (HS-1219). Written informed consent was obtained from the patient.

\section{Discussion}

\section{Comparison between dermoid cyst and liposarcoma in clinical and imaging features}

Dermoid cyst, also known as mature cystic teratoma, are benign tumors derived from embryonal tissue and are composed of somatic cell types from only one germ layer (1). After complete surgical excision, the overall 5-year survival rate is nearly $100 \%$ (4). However, liposarcoma is highly malignant, with an overall 5 -year survival rate of only 30 $60 \%$. For liposarcoma that is difficult to achieve negative resection margin ( $\mathrm{R} 0$ and $\mathrm{R} 1$ ), which is a crucial prognostic factor, preoperative chemoradiotherapy is recommended according to NCCN guidelines (5). If the margin is positive, reoperation or adjuvant radiotherapy is necessary (5). It was reported that the recurrence interval of liposarcoma after operation is longer in professional retroperitoneal tumor treatment center (6). Therefore, transferring to higher authority hospital was constantly suggested for this patient, and poor prognosis was also informed. Early recognition is challenging, but has important significance for timely treatment, surgical preparation, prognosis evaluation, and preoperative education of the patient.

According to imaging findings, it is challenging to differentiate a dermoid cyst without calcification and other classic signs from liposarcoma in retroperitoneal space. Firstly, both dermoid cyst and liposarcoma can be regular and well-encapsulated (6). Secondly, both of them are composed of mainly fatty material, with possible mixed and various densities. Calcification, mostly bone components which originate from mesoderm $(7,8)$, exits in almost $50 \%$ of teratomas (2). But it is rare in dermoid cysts which are exodermal. In liposarcoma, calcification can also be observed due to a variety of components like fibrosis, hemorrhage and necrosis (9). More importantly, in dermoid cysts, there can be thin and uniform separations (7), rather than linear, localized and irregular high density in liposarcoma (6). It is significant for differentiation. Thirdly, the soft tissue in benign dermoid cysts can also show an increased ${ }^{18} \mathrm{~F}$-FDG uptake in positron emission tomography (PET)/computed tomography (CT) (10). It may be attributed to the glucose utilization as the energy source for the active epithelium secretion (10).

In this case, the three-dimensional reconstruction CT showed obvious enlargements of reproductive veins, suggesting that the mass might be developed from the reproductive system. However, it did not get enough attention due to the formed first impression of liposarcoma in the early stage of admission.

\section{Clinical features of retroperitoneal dermoid cyst}

Mature cystic teratomas contains at least two germ cell layers. Dermoid cysts, containing only one germ layer, are very rare (7). Most retroperitoneal dermoid cysts reported were developed from at least two germ cell layers, but were also called mature cystic teratomas. The difference of the two diseases is yet to be clarified.

Conclusive data about dermoid cysts is insufficient, and most of them are reported as mature cystic teratomas. They are frequently characterized by a bimodal peak in incidence, occurring in the first 6 months of life and in early adulthood (7). Only $10-20 \%$ of them occur in adults more than 30 years of age (7). Retroperitoneal ones were usually misdiagnosed as adrenal tumors (11-13), pancreatic cysts (14), gastric stromal tumor (15) and so on. The majority of cases were asymptomatic $(8,14)$, and the most common symptom was nonspecific pain $(7,16)$. Patients whose adrenals were compressed might be present with vertigo and hypertension $(13,16)$, making the diagnosis more confusing. The incidence of malignant transformation is approximately $3-6 \%$ (12). Besides squamous cell carcinoma which accounts for $75-87 \%$ (17), enteric differentiation (11), mucinous adenocarcinoma (18), sarcoma, melanoma (9) and ganglion neuroblastoma (16) were also reported. There was a retroperitoneal recurrence after the ovary dermoid cyst resection (18).

\section{Histopathological analysis of dermoid cyst and the differential diagnoses}

Dermoid cyst, epidermoid cyst and mature cystic teratoma are likely to be confused (1) (Table 1). In ICD-10 coding, the first two are dominated by "cysts", while the last one is dominated by "teratoma" (1). It was shown in 2019 that in a hospital, $3.9 \%$ of the ICD-10 coding of these three diseases was wrong (1). The pathological characteristics are of vital importance to differentiation. For dermoid cysts and epidermoid cysts, both of them are lined 
Table 1 Comparison of dermoid cyst, epidermoid cyst and mature cystic teratoma

\begin{tabular}{|c|c|c|c|}
\hline & Dermoid cyst & Epidermoid cyst & Mature cystic teratoma \\
\hline \multicolumn{4}{|l|}{ ICD-coding } \\
\hline Dominated word & Cysts & Cysts & Teratoma \\
\hline Index & Ovary-dermoid (M9084/0)D27 & Skin-epidermis L72.0 & Adult (cyst) (M9080/0)-tumor, benign \\
\hline Cyst wall & Squamous epithelium, No scolex & Epidermis & Squamous epithelium, with scolex \\
\hline Lumen & $\begin{array}{l}\text { Skin appendages: sebaceous } \\
\text { glands, hair follicles, hair }\end{array}$ & $\begin{array}{l}\text { Keratinized materials, no skin } \\
\text { appendages }\end{array}$ & $\begin{array}{l}\text { Multiple germ layer components } \\
\text { (entoderm, mesoderm, ectoderm) }\end{array}$ \\
\hline Calcification & Rare & No & Common \\
\hline
\end{tabular}

ICD, International Classification of Diseases.

with squamous epithelium. However, there are no skin appendages such as sebaceous glands, hair follicles, and hair only in epidermoid cyst. For mature cystic teratomas and dermoid cysts, the former contains at least two germ cell layers with a common appearance of calcification $(12,14,17)$, while the latter is a single layer with rare calcification (7) and no scolex (1). Both of them are mainly composed of skin and skin appendages, so many clinicians used "mature cystic teratomas" to call them both (10-13) (Table 1). However, there is obvious difference in pathology and ICD-10 coding because of the number of germ cell layers and the presence of scolex (1).

\section{Treatment of dermoid cyst}

In the treatment of dermoid cysts, there were 7 cases reported to have successful results with sclerotherapy (3). However, the content is often too pultaceous to aspirate, and the dermal component of the cyst wall still exists, implying a risk of cyst re-expansion (3). Sclerotherapy can thus be used for patients who cannot undergo operation, or as preoperative therapy to reduce the tumor volume (3). Teratomas are largely resistant to radiotherapy and chemotherapy, which are only used for those with malignant features (19). Surgical removal is the mainstay of treatment due to a potential risk of malignancy $(3,10-12)$, and longterm follow-up is required. The number of veins and organs displaced and compressed by retroperitoneal mass is positively related to perioperative complications (20).

In this patient with retroperitoneal dermoid cyst, the surgical treatment was effective, safe and minimally invasive. Firstly, the tumor was behind the superior mesenteric arteriovenous. The angle of operation and surgical field of laparoscope had advantages in precisely undermining the posterior of the neoplasm where no major vessels go through. Secondly, the left margin of the tumor was closely related to the left renal hilum. Open surgery with a vertical incision on the left was then selected, in order to better expose and protect the integrity of the left kidney. More importantly, for large and transverse tumors, a traditional transverse or "inverted $\mathrm{Y}$ " incision is generally chosen. We believe the verticle left incision we performed could be minimally invasive in terms of injury of rectus abdominis, and was beneficial for patient's recovery.

In conclusion, dermoid cyst is a kind of mature cystic teratoma that contains only one germ cell layer. Retroperitoneal dermoid cyst is rare in adults. The purpose of this case was to present a rare case of retroperitoneal dermoid cyst which lacked the classical characteristics of teratomas in imaging, and mimicked a liposarcoma. A broad differential diagnosis is supposed to be made when evaluating a retroperitoneal fatty mass. For retroperitoneal dermoid cyst, calcification is rare, while thin and uniform separations are common.

\section{Acknowledgments}

Funding: None.

\section{Footnote}

Reporting Checklist: The authors have completed the CARE reporting checklist. Available at https://dx.doi. org/10.21037/gs-21-65 
Conflicts of Interest: All authors have completed the ICMJE uniform disclosure form (available at https://dx.doi. org/10.21037/gs-21-65). The authors have no conflicts of interest to declare.

Ethical Statement: The authors are accountable for all aspects of the work in ensuring that questions related to the accuracy or integrity of any part of the work are appropriately investigated and resolved. The study was approved by Ethics Committee of Peking Union Medical College Hospital (HS-1219). All procedures performed in studies involving human participants were in accordance with the ethical standards of the institutional and/or national research committee(s) and with the Helsinki Declaration (as revised in 2013). Written informed consent was obtained from the patient.

Open Access Statement: This is an Open Access article distributed in accordance with the Creative Commons Attribution-NonCommercial-NoDerivs 4.0 International License (CC BY-NC-ND 4.0), which permits the noncommercial replication and distribution of the article with the strict proviso that no changes or edits are made and the original work is properly cited (including links to both the formal publication through the relevant DOI and the license). See: https://creativecommons.org/licenses/by-nc-nd/4.0/.

\section{References}

1. Shu W, Ma Z. ICD-10 coding for Mature Cystic Teratomas and Dermoid and Epidermoid Cysts. Chinese Medical Record 2019;20:26-28.

2. O'Mara N, Cheung C, Mcmanus R, et al. Devil within: an incidental and rare finding of an extragonandal retroperitoneal germ cell teratoma carcinoid tumour. BMJ Case Rep 2018;2018:bcr-2017-223336.

3. Kitazawa T, Shiba M, Nagaya H, et al. Cranial dermoid cyst with long-term development treated by ethanol sclerotherapy: a case report. Case Reports Plast Surg Hand Surg 2020;7:130-3.

4. Pinson CW, ReMine SG, Fletcher WS, et al. Long-term Results With Primary Retroperitoneal Tumors. Arch Surg 1989;124:1168-73.

5. Mehren MV, Randall RL, Benjamin RS, et al. Soft Tissue Sarcoma, Version 2.2016, NCCN Clinical Practice
Guidelines in Oncology. J Natl Compr Canc Netw 2016;14:758-86.

6. Messiou C, Moskovic E, Vanel D, et al. Primary retroperitoneal soft tissue sarcoma: Imaging appearances, pitfalls and diagnostic algorithm. Eur J Surg Oncol 2017;43:1191-8.

7. Hoang VT, Trinh CT, Le TB, et al. Recurrence of retroperitoneal mature cystic teratoma in an adult: A case report. Radiol Case Rep 2019;14:692-6.

8. Sloan M, Fantus RJ, Paner GP, et al. Perineal dermoid cyst in a young male. Urol Case Rep 2020;33:101358.

9. Mantas D, Garmpis N, Polychroni D, et al. Retroperitoneal sarcomas: from diagnosis to treatment. Case series and review of the literature. G Chir 2020;41:18-33.

10. Lin SC, Yen RF, Chen YK. Dermoid cyst with secretion of CA 19-9 detected by 18F-FDG PET/CT: A case report. Medicine (Baltimore) 2020;99:e18988.

11. Sasi W, Ricchetti GA, Parvanta L, Carpenter R. Giant mature primary retroperitoneal teratoma in a young adult: report of a rare case and literature review. Case Rep Surg 2014;2014:930538.

12. Okulu E, Ener K, Aldemir M, et al. Primary Mature Cystc Teratoma Mimickng an Adrenal Mass in an Adult Male Patient. Korean J Urol 2014;55:148-51.

13. Tang DD, Zhang XS, Hao ZY, et al. A giant primary retroperitoneal mature cystic teratoma in right adrenal region in a 39-year-old female. Int J Clin Exp Med 2014;7:1611-3.

14. Peyvandi H, Arsan F, Alipour-Faz A, et al. Primary retroperitoneal mature cystic teratoma in an adult: A case report. Int J Surg Case Rep 2016;28:285-8.

15. Tiu A, Sovani V, Khan N, et al. Primary retroperitoneal mature cystic teratoma (dermoid cyst) in a 51-year-old male:Case report and historical literature review. SAGE Open Med Case Rep 2017;5:2050313X17700745.

16. Chen J, Khiyami A, Mchenry C. Retroperitoneal cystic teratoma masquerading as an incidentally discovered adrenal mass. Endocr Pract 2011;17:e130-4.

17. Huang PW, Liu YL, Miser JS, et al. Carcinoid tumor arising from a retroperitoneal mature cystic teratoma. Pediatr Neonatol 2018;59:520-2.

18. Cheung WL, Cao D. Colonic-type adenocarcinoma arising in a primary retroperitoneal mature cystic teratoma. Pathol Int 2008;58:792-6. 
19. Gatcombe HG, Vasily Assikis MD, David Kooby MD, et al. Primary retroperitoneal teratomas: a review of the literature. J Surg Oncol 2005;86:107-13.

Cite this article as: Ma X, Xiao J, Wang W. Retroperitoneal dermoid cyst mimicking a liposarcoma based on imaging assessment: case report and literature review. Gland Surg 2021;10(6):2062-2068. doi: 10.21037/gs-21-65
20. Liu Y, Hao X, Lu H, et al. Factors Associated With Perioperative Complications in the Treatment of Pediatric Retroperitoneal Teratoma. J Surg Res 2021;259:458-64. 